\title{
Human Epidermal Growth Factor Receptor-2 (HER2) expression in gastric adenocarcinoma and relevant clinicopathological features in a cohort of Sri Lankan patients
}

\author{
D. Subasinghe ${ }^{1}$, S. Sivaganesh ${ }^{2}$, A. Samarasekera ${ }^{3}$, M. P. Kumarasinghe ${ }^{4}$, D. N. Samarasekera ${ }^{5}$ \\ and M. D. S .Lokuhetty ${ }^{6}$
}

University Surgical Unit, National Hospital of Sri Lanka ${ }^{1.2 .5}$; Department of Pathology, National Hospital of Sri Lanka ${ }^{3}$; PathWest Laboratory Medicine, Perth, \&School of Pathology and Laboratory Medicine, University of Western Australia, Perth ${ }^{4}$; Department of Pathology, Faculty of Medicine, University of Colombo, Sri Lanka ${ }^{6}$

DOI: http://doi.org/10.4038/jdp.v11i2.7716

\section{Introduction}

Human epidermal growth factor receptor -2 (HER2) gene over-expression heralds adverse prognosis in gastric adenocarcinoma (GCa). HER2 protein over-expression by immunohistochemistry (IHC) ranges from 10\%-22.8\% and $11.7 \%-15.74 \%$ in Western and Asian studies respectively. We aimed to evaluate HER2 positivity in GCa by IHC and to study relevant clinicopathological relationships, as information is locally unavailable.

\section{Methodology}

Hundred consecutive GCa (excluding gastrooesophageal junction tumours) were recruited prospectively from the National Hospital of Sri Lanka over two years. The diagnosis was made on endoscopic biopsy/gastrectomy specimens. HER2 was assessed in paraffinized tumour using c-erB-2 oncoprotein (DAKO A0485) and Real-TM Envision system. Scores of $2+$ and $3+$ were considered positive, using standard HER2 expression criteria. Relationship between HER2 and clinicopathological parameters were analyzed using SPSS-21 and Chi-squared test.

\section{Results}

Study included 44 gastrectomies and 56 biopsies. Mean age was 61.1 years (range 3282). HER2 was positive in 9 (9\%) GCas: $2+$ in $3(5.4 \%)$ biopsies and $3(6.8 \%)$ resections, $3+$ in $1(1.8 \%)$ biopsy and $2(4.5 \%)$ resections. Eight (88.9\%) HER2 positive GCa occurred in $<60$ years with a male predominance $(n=7,77.7 \%)$. HER2 was positive in $4 / 58$ (6.9\%) proximal GCa and 5/42(11.9\%) distal GCa ( $P=0.48)$. HER2 positivity was $4(6.7 \%), 4(14.8 \%)$ and $1(7.1 \%)$ in intestinal, diffuse and mixed histological types respectively. Six (66.7\%) HER2 positive GCa presented at higher stage $(>=T 3)(p=0.31)$. HER2 positive cases showed, poor differentiation $(p=0.047)$, higher nuclear grade $(p=0.029)$, mitotic counts $>5 / \mathrm{HPF}$ $(p=0.003)$, extracellular mucin $(p=0.05)$, tumour necrosis $(p=0.037)$, perineural invasion ( $p=0.002)$ and nodal metastasis $(p=0.04)$ assessed only in gastric resections.

\section{Discussion and conclusion}

HER2 positivity with immunohistochemistry was $9 \%$ in the gastric carcinoma cohort and were associated with higher stage, adverse histological parameters and nodal metastasis in gastric resections. Adverse histological features can be used as screening features for HER2 testing in gastric carcinoma in limited resource settings. 